\title{
The impact of comorbid impulsive/compulsive disorders in problematic Internet use
}

\author{
SAMUEL R. CHAMBERLAIN ${ }^{1,2}$, KONSTANTINOS IOANNIDIS ${ }^{1,2}$ and JON E. GRANT ${ }^{3}$ \\ ${ }^{1}$ Department of Psychiatry, University of Cambridge, Cambridge, UK \\ ${ }^{2}$ Cambridge and Peterborough NHS Foundation Trust, Cambridge, UK \\ ${ }^{3}$ Department of Psychiatry and Behavioral Neuroscience, University of Chicago, Chicago, IL, USA
}

(Received: November 6, 2017; revised manuscript received: March 21, 2018; accepted: March 25, 2018)

\begin{abstract}
Background and aims: Problematic Internet use (PIU) is commonplace but is not yet recognized as a formal mental disorder. Excessive Internet use could result from other conditions such as gambling disorder. The aim of the study was to assess the impact of impulsive-compulsive comorbidities on the presentation of PIU, defined using Young's Diagnostic Questionnaire. Methods: A total of 123 adults aged 18-29 years were recruited using media advertisements, and attended the research center for a detailed psychiatric assessment, including interviews, completion of questionnaires, and neuropsychological testing. Participants were classified into three groups: PIU with no comorbid impulsive/compulsive disorders $(n=18)$, PIU with one or more comorbid impulsive/compulsive disorders $(n=37)$, and healthy controls who did not have any mental health diagnoses $(n=67)$. Differences between the three groups were characterized in terms of demographic, clinical, and cognitive variables. Effect sizes for overall effects of group were also reported. Results: The three groups did not significantly differ on age, gender, levels of education, nicotine consumption, or alcohol use (small effect sizes). Quality of life was significantly impaired in PIU irrespective of whether or not individuals had comorbid impulsive/compulsive disorders (large effect size). However, impaired response inhibition and decision-making were only identified in PIU with impulsive/compulsive comorbidities (medium effect sizes). Discussion and conclusions: Most people with PIU will have one or more other impulsive/ compulsive disorders, but PIU can occur without such comorbidities and still present with impaired quality of life. Response inhibition and decision-making appear to be disproportionately impacted in the case of PIU comorbid with other impulsive/compulsive conditions, which may account for some of the inconsistencies in the existing literature. Large scale international collaborations are required to validate PIU and further assess its clinical, cognitive, and biological sequelae.
\end{abstract}

Keywords: compulsivity, impulsivity, cognition, Internet

\section{INTRODUCTION}

The Internet has gone from being a narrowly available technology in the 1980 s to constituting an all-pervasive aspect of society in the present day. At least $90 \%$ of young adults use the Internet in the USA, Europe, and Asia (Durkee et al., 2012; Kuss, Griffiths, Karila, \& Billieux, 2014). Its availability is rapidly increasing in other parts of the world such as in Africa. While there are positive spects of the Internet, such as rapid and convenient availability of information, it is recognized that some people develop a maladaptive use of the Internet, spending a large amount of time online and neglecting other areas of life. Problematic Internet use (PIU) is a putative entity not yet recognized by diagnostic classification systems, but which has received growing research and clinical attention (Bernardi \& Pallanti, 2009; Weinstein \& Lejoyeux, 2010). PIU has been strongly associated with mental disorders [depressive and anxiety disorders, attention-deficit hyperactivity disorder (ADHD)] (Ho et al., 2014) and impaired functioning
(Derbyshire et al., 2013). Depending on the precise operational definition used, the prevalence of PIU has been estimated to be $1 \%-38 \%$ in young people (Durkee et al., 2012).

Whether or not PIU should be regarded as a formal mental health disorder remains contentious (Demetrovics \& Griffiths, 2012; Przybylski, Weinstein, \& Murayama, 2017). In part, this may not only reflect the relative newness of the associated behaviors (due to the Internet only being developed in the 1980s) but also concern regarding overpathologizing human behavior, and whether to focus on a particular type of online behavior (e.g., Internet gaming disorder) (Griffiths et al., 2016; Kiraly \& Demetrovics, 2017; Kuss \& Billieux, 2017) or many, as would be suggested by maladaptive Internet use severity correlating

* Corresponding author: Dr. Samuel R. Chamberlain, MD, PhD; Department of Psychiatry, University of Cambridge, Box 189 Level E4, Addenbrooke's Hospital, Cambridge CB2 0QQ, UK; E-mail: src33@cam.ac.uk

This is an open-access article distributed under the terms of the Creative Commons Attribution-NonCommercial 4.0 International License, which permits unrestricted use, distribution, and reproduction in any medium for non-commercial purposes, provided the original author and source are credited, a link to the CC License is provided, and changes - if any - are indicated. 
with a range of online behaviors (Ioannidis et al., 2016, 2017). Another important issue is whether PIU simply reflects other underlying mental health disorders (Kuss \& Billieux, 2017). For example, if a person uses the Internet excessively to gamble or shop, this may reflect gambling disorder or compulsive buying disorder respectively, and if they use it for compulsive sexual acts, this may reflect compulsive sex behavior disorder. It has also been noted that formal mental health disorders, including ADHD, mood, and anxiety disorders, substance use disorders, commonly occur in PIU (odds ratios of about three per disorder in a meta-analysis of the literature) (Ho et al., 2014). In these cases, excessive Internet use could be a consequence or counterbalancing act due to the presence of a well-known mental illness (e.g., online social contact to counteract social phobia).

Cognitive dysfunction relating to decision-making, executive function (e.g., set-shifting difficulties), and impulse control have been implicated in the context of other behavioral addictions and thus may also be relevant for PIU (Chamberlain et al., 2016; Leeman \& Potenza, 2013; Smith, Mattick, Jamadar, \& Iredale, 2014). However, studies of cognition in PIU are few in number and so far have yielded quite contradictory findings, even relative to those for other behavioral and substance addictions (Smith et al., 2014). There are many potential reasons for this, including the use of non-standard cognitive tests, different operationalizations of PIU, and failure to control for comorbidities notably ADHD (Ho et al., 2014), which itself is associated with marked cognitive impairment in certain of these cognitive domains (Chamberlain et al., 2010). Similarly, existing cognitive studies did not typically rule out other underlying impulsive-compulsive disorders, such as gambling disorder, hair-pulling disorder, compulsive buying disorder, compulsive sex behavior disorder, compulsive stealing (kleptomania), or obsessive-compulsive disorder (OCD).

Therefore, the aim of this study was to compare demographic, clinical, and cognitive measures between three groups: those with PIU who did not have any potentially contributing comorbid impulsive/compulsive disorders, those with PIU who had such comorbidities, and healthy controls who did not have any mental health diagnoses. We hypothesized that PIU without impulsive/compulsive comorbidities would occupy an intermediate position between healthy controls and PIU comorbid with impulsive/compulsive disorders in terms of impaired quality of life, cognitive function, and scores on relevant trait questionnaires.

\section{METHODS}

\section{Participants}

A total of 123 individuals aged 18-29 years were recruited using media advertisements in a US city. The only inclusion criterion was gambling at least five times in the preceding year, as the study was part of a broader project examining gambling in young adults. As such, this can be seen as an enriched sample. The only exclusion criterion was an inability to understand/undertake the assessments. Participants provided written informed consent after receiving a complete description of the study, and attended the study center to complete a face-to-face structured psychiatric interview, questionnaires, and cognitive testing using a touch-screen computer.

\section{Assessments}

Validated screening tools for PIU are underresearched. We opted to use Young's Diagnostic Questionnaire (YDQ; Young, 2009) to identify PIU, because it is convenient to administer. The YDQ is an eight-item set of questions, which was derived from prior criteria for substance-use disorder and pathological gambling, but applied to maladaptive Internet use. The YDQ considers preoccupation with the Internet, escalating quantities of time spent using the Internet, repeated unsuccessful attempts to cut back, restlessness/irritability when attempting to cut back, staying online longer than intended, jeopardizing careers/ scholarship/relationships, lying to others, and using the Internet to escape from life or emotional difficulties. Thus, the YDQ captures a broad range of PIU thoughts and behaviors. Problematic Internet addiction was defined as endorsing four or more of these symptoms over the preceding 12-month period, based on the number of criteria often used for gambling disorder, from which this instrument was partly derived, but accounting for YDQ having fewer total items. It should be noted that our definition of "PIU" identifies people with relatively more problems, rather than constituting a formal mental disorder diagnosis, because such a diagnosis (and its definition) is not yet listed in psychiatric classification systems.

Presence of current psychiatric disorders was evaluated using the Mini International Neuropsychiatric Inventory (MINI; Sheehan et al., 1998), the Minnesota Impulse Disorder Inventory (MIDI; Grant, Levine, Kim, \& Potenza, 2005), and the World Health Organization Adult ADHD Self-Report Scale (ASRS v1.1; Kessler et al., 2005, 2007). The MINI identifies mainstream psychiatric disorders, such as mood and anxiety disorders (including OCD), whereas the MIDI identifies impulse control disorders (including gambling disorder, compulsive sex behavior disorder, hair-pulling disorder, skin-picking disorder, kleptomania, pyromania, intermittent explosive disorder, and compulsive buying). The MINI and MIDI were completed by a trained assessor. The ASRS is a selfcomplete questionnaire, which yields a total score; total score on Part A was used to determine presence or absence of ADHD using the previously published threshold, which yields extremely high classification accuracy (Kessler et al., 2005).

Participants also completed the following questionnaires: Quality of Life Inventory (QOLI) to measure satisfaction in multiple domains (Frisch, 1998), the Barratt Impulsiveness Questionnaire (v11) to measure impulsive personality traits (Stanford et al., 2016), and the Padua Inventory to measure obsessive-compulsive traits (Burns, Keortge, Formea, \& Sternberger, 1996; Sanavio, 1988). We also collected relevant background information, including age, gender, educational level, frequency of alcohol use, and numbers of cigarette packs smoked per day. 
Cognitive testing was conducted using three computerized tests from the Cambridge Neuropsychological Test Automated Battery (CANTABeclipse, version 3, Cambridge Cognition Ltd., UK). Based on existing models of behavioral addictions (Chamberlain et al., 2016; Clark, 2010; Dong \& Potenza, 2014), we focused on inhibitory control, decisionmaking, and set-shifting.

Inhibitory control was measured using the Stop-Signal Task (SST; Aron, Robbins, \& Poldrack, 2014; Logan, Cowan, \& Davis, 1984). On the SST, a series of directional arrows were presented on the computer screen one at a time, and volunteers made quick responses depending on the direction of arrows (left button for left-facing arrow and right button for right-facing arrow). On some trials, an auditory stop signal ("beep") occurred a variable time after presentation of the go cue, indicating that the volunteer should attempt to omit a response for the given trial. By dynamically modulating the time between presentation of the arrow and the stop signal, the task calculated the stop-signal reaction time - a measure of time taken to suppress a response that would normally be made. Longer stop-signal reaction times equate to worse top-down control.

Decision-making was measured using the Cambridge Gamble Task (CGT; Rogers et al., 2003). On each trial, 10 boxes were shown, some blue and some red, with a token having been hidden behind one of these. The participant selected the color of the box they believed a token was hidden behind, and then decided how many points to gamble on having made the correct decision. The main measures of decision-making on the task were the proportion of points gambled overall, the proportion of rational decisions made (proportion of trials when the volunteer opted for the color that was in the majority), and the extent of risk adjustment (the extent to which individuals modulated the amount gambled depending on the probability of making correct choices).

Set-shifting was assessed using the Intra-Dimensional/ Extra-Dimensional set-shift task (IED; Birrell \& Brown, 2000; Owen, Roberts, Polkey, Sahakian, \& Robbins, 1991). This paradigm is based on the Wisconsin Card Sorting Task but decomposes different aspects of rule acquisition and flexible responding over nine task stages. Volunteers choose between two stimuli presented on the computer screen on each trial, and receive feedback as to whether their choice was "right" or "wrong." Through trial and error, the volunteer attempts to learn a rule about which stimulus is correct. The computer alters this underlying rule when the current rule has been learnt by the volunteer. The main measure on the task is the total number of errors made, adjusted for stages that were failed. Where this composite measure is statistically significant for a comparison of interest, then scores on individual task stages can be explored to confirm the main cognitive problems driving the overall impairment on the task.

\section{Data analysis}

The participants were grouped into three categories: those with PIU who did not have comorbid impulsive/compulsive disorders (OCD, ADHD, gambling disorder, compulsive sex behavior disorder, hair-pulling disorder, skin-picking disorder, kleptomania, pyromania, intermittent explosive disorder, or compulsive buying), those who had one or more of such comorbidities, and healthy controls who did not have any mental health diagnoses. Demographic, clinical, and cognitive characteristics of the three study groups were tabulated and compared using one-way analysis of variance (ANOVA) or $\chi^{2}$ tests as appropriate. ANOVA was used for continuous variables fulfilling normality assumptions, whereas $\chi^{2}$ was used for categorical variables. Effect sizes were also reported for the overall effects of group, to give an indication of possible clinical significance $\left(\eta^{2}\right.$ for ANOVA and $\phi$ for $\chi^{2}$ tests). By convention for $\eta^{2}, 0.01$ is a small effect, 0.06 a medium effect, and 0.14 a large effect; and for $\phi, 0.1$ is a small effect, 0.3 a medium effect, and 0.5 a large effect. Where the main effect of group was significant, this was further explored using paired post-hoc $t$-tests or alternative tests as indicated. This being an exploratory study, statistical significance was defined as $p<.05$ uncorrected, two-tailed. Data were analyzed using JMP Pro version 13 .

\section{Ethics}

The participants provided written informed consent after receiving a complete description of the study. This research was approved by an Institutional Review Board.

\section{RESULTS}

Of the 123 young adults, 55 (44.7\%) endorsed symptoms consistent with PIU. The sample comprised participants with PIU who were not comorbid with impulsive/compulsive disorders $(n=18)$, participants who had PIU with one or more comorbid impulsive/compulsive disorders $(n=37)$, and healthy controls $(n=67)$ (i.e., no major mental disorder detected based on clinical interview including the MINI and MIDI). The impulsive/compulsive disorders in the comorbid group were $[N(\%)]$ : compulsive buying disorder $4(10.8 \%)$, intermittent explosive disorder 6 (16.2\%), gambling disorder $21(56.8 \%)$, compulsive sexual behavior disorder $4(10.8 \%)$, binge-eating disorder $3(8.1 \%)$, skin-picking disorder 4 (10.8\%), OCD 2 (5.4\%), and ADHD 17 (45.9\%).

As can be seen in Table 1, the three groups did not significantly differ in terms of age, gender, education level, nicotine consumption, or alcohol use. The two PIU groups did not significantly differ for the number of YDQ items endorsed [PIU mean $(S D), 5.2(0.3)$ items; PIU with impulsive/ compulsive disorders, $5.4(0.2)$; $t$-test, $t=0.27 ; p=.42]$.

Quality of life on the QOLI (Frisch, 1998) was significantly impaired in both PIU groups compared with healthy controls, and PIU groups did not significantly differ from each other on quality of life. Approximately, $60 \%-65 \%$ of PIU participants had comorbid mental disorders that were not impulsive/compulsive (e.g., depression and anxiety) according to the MINI, and the two groups did not significantly differ for this rate.

For the personality questionnaires, significantly increased Padua obsessive-compulsive scores were found in both PIU groups versus the controls, whereas only the PIU cases with comorbid impulsive/compulsive disorders had 
Chamberlain et al.

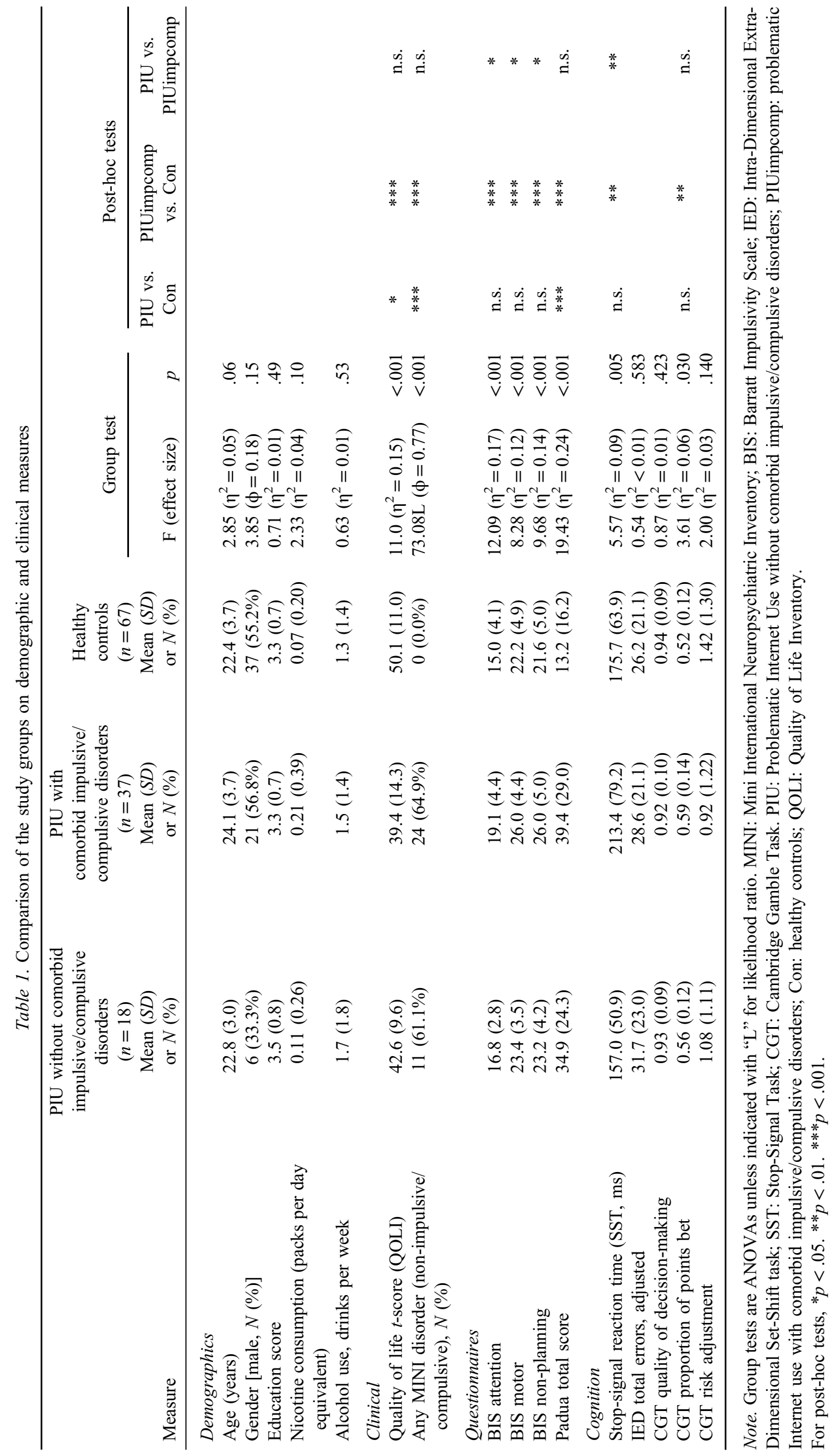


significantly elevated Barratt impulsiveness scores on all three subscales (motor, non-planning, and attentional impulsiveness) compared with the controls. The PIU group with comorbid impulsive/compulsive disorders had significantly higher Barratt scores than the pure PIU cases, whereas the two PIU groups did not significantly differ from each other for Barratt impulsiveness scores.

In terms of cognitive performance, the PIU participants with comorbid impulsive/compulsive disorders showed significant stop-signal impairment and decision-making (proportion of points bet) impairment compared with the controls, whereas pure PIU cases did not significantly differ from the healthy controls. PIU with comorbid impulsive/ compulsive disorders had significant stop-signal impairment versus the other PIU group, but these two groups did not differ from each other significantly for proportion of points bet. There were neither main effects of group on other task measures from the gamble task nor for the set-shifting task.

\section{DISCUSSION AND CONCLUSIONS}

In this study, we assessed PIU in a sample recruited from a large US city through media advertisements. Our aim was to clarify whether the profile of PIU was influenced by presence or absence of co-occurring impulsive and compulsive disorders. Several studies exploring demographic, clinical, and cognitive measures in PIU have not considered the impact of such comorbid conditions (e.g., ADHD and gambling disorder). The key finding here was that quality of life was impaired in people with PIU (large effect size), even in the absence of comorbid impulsive/compulsive disorders. Both PIU groups had mean quality of life in the low range, significantly lower than the healthy control group, whose mean quality of life was in the normal range. These results suggest that PIU can occur in the absence of such impulsive/ compulsive conditions, i.e., it is not always purely a conduit. The most common comorbid impulsive/compulsive disorders in the comorbid PIU group were gambling disorder and ADHD, although a broad spread of other conditions was also observed. It should be noted that the sample was somewhat enriched for gambling symptomatology.

The finding that the majority of PIU participants recruited in this study had one or more impulsive/compulsive disorders is in keeping with a previous meta-analysis in PIU as pertains to ADHD (Ho et al., 2014) as well as with the notion that comorbid behavioral addictions (e.g., gambling disorder) can contribute to this condition. Nonetheless, our findings militate against disregarding PIU in its own right, as a substantial proportion of cases did not have such impulsive/compulsive comorbidities, and were also functionally impaired significantly and to a similar degree, with mean quality of life in the poor range. Furthermore, PIU cases without comorbid impulsive/compulsive problems had similarly elevated rates of other mental disorders (those not related to impulsivity and compulsivity) compared with the other PIU group, suggesting that its clinical associations are not benign.

Intriguingly, compared with the healthy controls, only the PIU group with comorbid impulsive/compulsive disorders had significantly impaired response inhibition (stop-signal test; medium effect size), gambled more points (CGT; medium effect size), and had elevated personality traits of impulsiveness (Barratt Questionnaire; large effect size). Some caution is needed when interpreting these findings as the PIU group without comorbid impulsive/ compulsive disorders had a smaller sample size.

These results suggest that impaired performance on inhibitory control tasks reported in elements of the PIU literature could have stemmed in part from the impact of comorbid impulsive or compulsive symptoms. This may help to explain, in part, why inconsistencies have been found on inhibition tasks in PIU, as noted in a systematic review (Smith et al., 2014). In contrast, we found that obsessive-compulsive tendencies as measured by the Padua Inventory were elevated in both PIU groups (large effect size). It is important to note that this did not stem from OCD at a categorical level, because none in our sample had OCD based on the M.I.N.I. interview. PIU can be seen as being relatively compulsive from a trait personality point of view, but we did not find set-shifting impairment on the IED; hence, this compulsivity does not seem to reflect generalized attentional rigidity.

Several limitations should be noted for this study. We defined PIU using YDQ, which is a convenient short format derived from criteria for gambling and substance-use disorders but applied to pathological use of the Internet. We believe our choice of a score of 4 or more on YDQ was appropriate based on parallels with those approaches used in gambling-use disorder. Nonetheless, there are various ways of defining PIU and there is, to date, no consensus in the field as to which method constitutes the "gold-standard" or what cut-offs are optimal. For example, Young's Internet Use Questionnaire is longer and may be more useful for assessing severity as opposed to the diagnosis, and several other scales exist, or are in development. YDQ (and the related Internet Use Questionnaire) has received only limited psychometric validation since their inception, and may have an inconsistent factor structure (Kiraly, Nagygyorgy, Koronczai, Griffiths, \& Demetrovics, 2015). For these reasons, future work may prefer to use alternative scales that have received more comprehensive validation. For example, the short (6-item) version of the Problematic Internet Use Questionnaire appears to have good properties based on initial validation in a nationally representative sample of adolescents (Demetrovics et al., 2016). This was a relatively small cross-sectional study; as such, causality cannot be inferred, and the study was only powered to detect medium-large as opposed to small group differences. For this reason, we did not correct for multiplicity. No participants had a prior diagnosis of Autism based on clinical screening, but we did not include a dimensional measure of autistic spectrum disorder in our protocol. Our sample can be seen as enriched as participants were recruited on the basis of some level of gambling over the past year; hence, findings may differ in participants recruited without this criterion in other research. Finally, it remains to be seen whether the findings generalize to other populations, such as PIU presenting in treatment settings, more severe cases, or in other age groups.

In conclusion, this study found that while most people with PIU had one or more impulsive-compulsive disorders, 
quality of life was still impaired even in the group of PIU participants without these comorbidities (large effect size). However, there were differences in the presentation of PIU contingent on such comorbidities. PIU with comorbid impulsive/compulsive disorders had more marked abnormalities in response to inhibition and decision-making on the utilized cognitive tasks (medium effect sizes), and elevated trait impulsiveness on the Barratt Questionnaire (large effect size) than did the PIU group without such comorbidities. Both PIU groups showed elevations in dimensional compulsivity (Padua Inventory; large effect size). Future work should refine and arrive at a consensus regarding the definition of PIU and how best to identify it and should further explore the impact of impulsive and compulsive comorbidities on the presentation of this prevalent, putative mental disorder.

Funding sources: This research was supported by a grant from the National Center for Responsible Gaming to Dr. JEG and by a Wellcome Trust Clinical Fellowship Grant to Dr. SRC (reference no.: 110049/Z/15/Z). Dr. KI's research is supported by Health Education East of England Higher Training Special interest sessions. This article is based on work from COST Action (CA16207), supported by COST (European Cooperation in Science and Technology). The authors would like to thank Dr. Naomi Fineberg for providing feedback on a draft version of this manuscript, undertaken as part of the COST Action Network.

Authors' contribution: Dr. JEG developed the study protocol and undertook the data collection. Dr. SRC undertook the data analysis. All the authors substantially contributed in writing the manuscript and interpretation. SRC had access to all data from the study, both what is reported and what is unreported, and had complete freedom to direct its analysis and its reporting. He affirms that there was no external editorial direction or censorship.

Conflict of interest: Dr. JEG has received research grants from NIMH, NIDA, National Center for Responsible Gaming, and Takeda Pharmaceuticals. He had received yearly compensation from Springer Publishing for acting as Editor-in-Chief of the Journal of Gambling Studies and had received royalties from Oxford University Press, American Psychiatric Publishing, Inc., Norton Press, and McGraw Hill. Dr. SRC consults for Cambridge Cognition and Shire.

\section{REFERENCES}

Aron, A. R., Robbins, T. W., \& Poldrack, R. A. (2014). Inhibition and the right inferior frontal cortex: One decade on. Trends in Cognitive Sciences, 18(4), 177-185. doi:10.1016/j.tics. 2013.12.003

Bernardi, S., \& Pallanti, S. (2009). Internet addiction: A descriptive clinical study focusing on comorbidities and dissociative symptoms. Comprehensive Psychiatry, 50(6), 510-516. doi:10.1016/j.comppsych.2008.11.011
Birrell, J. M., \& Brown, V. J. (2000). Medial frontal cortex mediates perceptual attentional set shifting in the rat. Journal of Neuroscience, 20(11), 4320-4324. doi:10.1523/ JNEUROSCI.20-11-04320.2000

Burns, G. L., Keortge, S. G., Formea, G. M., \& Sternberger, L. G. (1996). Revision of the Padua Inventory of obsessive compulsive disorder symptoms: Distinctions between worry, obsessions, and compulsions. Behavior Research and Therapy, 34(2), 163-173. doi:10.1016/0005-7967(95)00035-6

Chamberlain, S. R., Lochner, C., Stein, D. J., Goudriaan, A. E., van Holst, R. J., Zohar, J., \& Grant, J. E. (2016). Behavioural addiction - A rising tide? European Neuropsychopharmacology, 26(5), 841-855. doi:10.1016/j.euroneuro.2015.08.013

Chamberlain, S. R., Robbins, T. W., Winder-Rhodes, S., Muller, U., Sahakian, B. J., Blackwell, A. D., \& Barnett, J. H. (2010). Translational approaches to frontostriatal dysfunction in attention-deficit/hyperactivity disorder using a computerized neuropsychological battery. Biological Psychiatry, 69(12), 1192-1203. doi:10.1016/j.biopsych.2010.08.019

Clark, L. (2010). Decision-making during gambling: An integration of cognitive and psychobiological approaches. Philosophical Transactions of the Royal Society of London. Series B, Biological Sciences, 365(1538), 319-330. doi:10.1098/rstb. 2009.0147

Demetrovics, Z., \& Griffiths, M. D. (2012). Behavioral addictions: Past, present and future. Journal of Behavioral Addictions, 1(1), 1-2. doi:10.1556/JBA.1.2012.1.0

Demetrovics, Z., Kiraly, O., Koronczai, B., Griffiths, M. D., Nagygyorgy, K., Elekes, Z., Tamás, D., Kun, B., Kökönyei, G., \& Urban, R. (2016). Psychometric properties of the Problematic Internet Use Questionnaire short-form (PIUQ-SF-6) in a nationally representative sample of adolescents. PLoS One, 11(8), e0159409. doi:10.1371/journal. pone.0159409

Derbyshire, K. L., Lust, K. A., Schreiber, L. R., Odlaug, B. L., Christenson, G. A., Golden, D. J., \& Grant, J. E. (2013). Problematic Internet use and associated risks in a college sample. Comprehensive Psychiatry, 54(5), 415-422. doi:10.1016/j.comppsych.2012.11.003

Dong, G., \& Potenza, M. N. (2014). A cognitive-behavioral model of Internet gaming disorder: Theoretical underpinnings and clinical implications. Journal of Psychiatric Research, 58, 7-11. doi:10.1016/j.jpsychires.2014.07.005

Durkee, T., Kaess, M., Carli, V., Parzer, P., Wasserman, C., Floderus, B., Apter, A., Balazs, J., Barzilay, S., Bobes, J., Brunner, R., Corcoran, P., Cosman, D., Cotter, P., Despalins, R., Graber, N., Guillemin, F., Haring, C., Kahn, J. P., Mandelli, L., Marusic, D., Mészáros, G., Musa, G. J., Postuvan, V., Resch, F., Saiz, P. A., Sisask, M., Varnik, A., Sarchiapone, M., Hoven, C. W., \& Wasserman, D. (2012). Prevalence of pathological Internet use among adolescents in Europe: Demographic and social factors. Addiction, 107(12), 2210-2222. doi:10.1111/j.1360-0443.2012. 03946.x

Frisch, M. B. (1998). Quality of life therapy and assessment in health care. Clinical Psychology: Science and Practice, 5, 19-40. doi:10.1111/j.1468-2850.1998.tb00132.x

Grant, J. E., Levine, L., Kim, D., \& Potenza, M. N. (2005). Impulse control disorders in adult psychiatric inpatients. American Journal of Psychiatry, 162(11), 2184-2188. doi:10.1176/appi. ajp.162.11.2184 
Griffiths, M. D., van Rooij, A. J., Kardefelt-Winther, D., Starcevic, V., Kiraly, O., Pallesen, S., Müller, K., Dreier, M., Carras, M., Prause, N., King, D. L., Aboujaoude, E., Kuss, D. J., Pontes, H. M., Lopez Fernandez, O., Nagygyorgy, K., Achab, S., Billieux, J., Quandt, T., Carbonell, X., Ferguson, C. J., Hoff, R. A., Derevensky, J., Haagsma, M. C., Delfabbro, P., Coulson, M., Hussain, Z., \& Demetrovics, Z. (2016). Working towards an international consensus on criteria for assessing Internet gaming disorder: A critical commentary on Petry et al. (2014). Addiction, 111(1), 167-175. doi:10.1111/add.13057

Ho, R. C., Zhang, M. W., Tsang, T. Y., Toh, A. H., Pan, F., Lu, Y., Cheng, C., Yip, P. S., Lam, L. T., Lai, C. M., Watanabe, H., \& Mak, K. K. (2014). The association between Internet addiction and psychiatric co-morbidity: A meta-analysis. $B M C$ Psychiatry, 14(1), 183. doi:10.1186/1471-244X-14-183

Ioannidis, K., Chamberlain, S. R., Treder, M. S., Kiraly, F., Leppink, E. W., Redden, S. A., Stein, D. J., Lochner, C., \& Grant, J. E. (2016). Problematic Internet use (PIU): Associations with the impulsive-compulsive spectrum. An application of machine learning in psychiatry. Journal of Psychiatric Research, 83, 94-102. doi:10.1016/j.jpsychires.2016.08.010

Ioannidis, K., Treder, M. S., Chamberlain, S. R., Kiraly, F., Redden, S. A., Stein, D. J., Lochner, C., \& Grant, J. E. (2017). Problematic Internet addiction as an age-related multifaceted problem: Evidence from a two-site survey. Addictive Behaviors, 81, 157-166. doi:10.1016/j.addbeh. 2018.02.017

Kessler, R. C., Adler, L., Ames, M., Demler, O., Faraone, S., Hiripi, E., Howes, M. J., Jin, R., Secnik, K., Spencer, T., Ustun, T. B., \& Walters, E. E. (2005). The World Health Organization Adult ADHD Self-Report Scale (ASRS): A short screening scale for use in the general population. Psychological Medicine, 35(2), 245-256. doi:10.1017/S00332917 04002892

Kessler, R. C., Adler, L. A., Gruber, M. J., Sarawate, C. A., Spencer, T., \& Van Brunt, D. L. (2007). Validity of the World Health Organization Adult ADHD Self-Report Scale (ASRS) Screener in a representative sample of health plan members. International Journal of Methods in Psychiatric Research, 16(2), 52-65. doi:10.1002/mpr.208

Kiraly, O., \& Demetrovics, Z. (2017). Inclusion of gaming disorder in ICD has more advantages than disadvantages. Journal of Behavioral Addictions, 6(3), 280-284. doi:10.1556/2006.6. 2017.046

Kiraly, O., Nagygyorgy, B. K., Koronczai, B., Griffiths, M. D., \& Demetrovics, Z. (2015). Assessment of problematic Internet use and online video gaming. In E. Aboujaoude \& V. Starcevic (Eds.), Mental health in the digital age (pp. 46-58). New York, NY: Oxford University Press.

Kuss, D. J., \& Billieux, J. (2017). Technological addictions: Conceptualisation, measurement, etiology and treatment. Addictive Behaviors, 64, 231-233. doi:10.1016/j.addbeh. 2016.04.005
Kuss, D. J., Griffiths, M. D., Karila, L., \& Billieux, J. (2014). Internet addiction: A systematic review of epidemiological research for the last decade. Current Pharmaceutical Design, 20(25), 4026-4052. doi:10.2174/13816128113199990617

Leeman, R. F., \& Potenza, M. N. (2013). A targeted review of the neurobiology and genetics of behavioural addictions: An emerging area of research. Canadian Journal of Psychiatry, 58(5), 260-273. doi:10.1177/070674371305800503

Logan, G. D., Cowan, W. B., \& Davis, K. A. (1984). On the ability to inhibit simple and choice reaction time responses: A model and a method. Journal of Experimental Psychology Human Perception \& Performance, 10(2), 276-291. doi:10.1037/ 0096-1523.10.2.276

Owen, A. M., Roberts, A. C., Polkey, C. E., Sahakian, B. J., \& Robbins, T. W. (1991). Extra-dimensional versus intradimensional set shifting performance following frontal lobe excisions, temporal lobe excisions or amygdalo-hippocampectomy in man. Neuropsychologia, 29(10), 993-1006. doi:10.1016/00283932(91)90063-E

Przybylski, A. K., Weinstein, N., \& Murayama, K. (2017). Internet gaming disorder: Investigating the clinical relevance of a new phenomenon. American Journal of Psychiatry, 174(3), 230-236. doi:10.1176/appi.ajp.2016.16020224

Rogers, R. D., Tunbridge, E. M., Bhagwagar, Z., Drevets, W. C., Sahakian, B. J., \& Carter, C. S. (2003). Tryptophan depletion alters the decision-making of healthy volunteers through altered processing of reward cues. Neuropsychopharmacology, 28(1), 153-162. doi:10.1038/sj.npp.1300001

Sanavio, E. (1988). Obsessions and compulsions: The Padua Inventory. Behaviour Research and Therapy, 26(2), 169-177. doi:10.1016/0005-7967(88)90116-7

Sheehan, D. V., Lecrubier, Y., Sheehan, K. H., Amorim, P., Janavs, J., Weiller, E., Hergueta, T., Baker, R., \& Dunbar, G. C. (1998). The Mini-International Neuropsychiatric Interview (M.I.N.I.): The development and validation of a structured diagnostic psychiatric interview for DSM-IV and ICD-10. Journal of Clinical Psychiatry, 59(Suppl. 20), 22-33; quiz 34-57. doi:10.1016/S0924-9338(97)83296-8

Smith, J. L., Mattick, R. P., Jamadar, S. D., \& Iredale, J. M. (2014). Deficits in behavioural inhibition in substance abuse and addiction: A meta-analysis. Drug and Alcohol Dependence, 145, 1-33. doi:10.1016/j.drugalcdep.2014.08.009

Stanford, M. S., Mathias, C. W., Dougherty, D. M., Lake, S. L., Anderson, N. E., \& Patton, J. H. (2016). Fifty years of the Barratt Impulsiveness Scale: An update and review. Personality and Individual Differences, 47(5), 385-395. doi:10.1016/j. paid.2009.04.008

Weinstein, A., \& Lejoyeux, M. (2010). Internet addiction or excessive Internet use. American Journal of Drug and Alcohol Abuse, 36(5), 277-283. doi:10.3109/00952990.2010.491880

Young, K. (2009). Internet addiction: The emergence of a new clinical disorder. CyberPsychology \& Behavior, 1(3), 237-244. doi:10.1089/cpb.1998.1.237 